\title{
Diacronie
}

Studi di Storia Contemporanea

$\mathrm{N}^{\circ} 32,4 \mid 2017$

Proiezioni individuali e agire collettivo nella storia

\section{Feminist sex wars}

Arianna Pasqualini

\section{(2) OpenEdition}

\section{Journals}

\section{Edizione digitale}

URL: http://journals.openedition.org/diacronie/6742

DOI: 10.4000/diacronie.6742

ISSN: 2038-0925

\section{Editore}

Association culturelle Diacronie

\section{Notizia bibliografica digitale}

Arianna Pasqualini, «Feminist sex wars », Diacronie [Online], №32, 4 | 2017, documento 7, Messo online il 29 décembre 2017, consultato il 02 mai 2019. URL : http://journals.openedition.org/diacronie/6742 ; DOI : 10.4000/diacronie.6742 


\section{Diacronie}

\section{Studi di Storia Contemporanea}

\section{$32,4 / 2017$}

Proiezioni individuali e agire collettivo nella storia. Ruoli sociali, aspetti politici e nodi storiografici tra pubblico e privato

\section{Feminist sex wars}

\section{Arianna PASQUALINI}

Per citare questo articolo:

PASQUALINI, Arianna, «Feminist sex wars», Diacronie. Studi di Storia Contemporanea : Proiezioni individuali e agire collettivo nella storia. Ruoli sociali, aspetti politici e nodi storiografici tra pubblico e privato, 32, 4/2017, 29/12/2017,

URL: < http://www.studistorici.com/2017/12/29/pasqualini_numero_32/ >

Diacronie Studi di Storia Contemporanea $\rightarrow$ http://www.diacronie.it

Rivista storica online. Uscita trimestrale.

redazione.diacronie@hotmail.it

Comitato di direzione: Naor Ben-Yehoyada - João Fábio Bertonha - Christopher Denis-Delacour - Maximiliano Fuentes Codera Anders Granås Kjøstvedt - John Paul Newman - Deborah Paci - Niccolò Pianciola - Spyridon Ploumidis - Wilko Graf Von Hardenberg

Comitato di redazione: Jacopo Bassi - Luca Bufarale - Gianluca Canè - Fausto Pietrancosta - Alessandro Salvador - Matteo Tomasoni - Luca Zuccolo

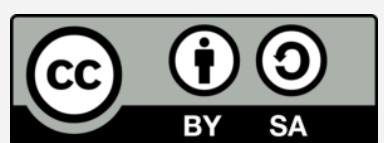

Diritti: gli articoli di Diacronie. Studi di Storia Contemporanea sono pubblicati sotto licenza Creative Commons 3.0. Possono essere riprodotti e modificati a patto di indicare eventuali modifiche dei contenuti, di riconoscere la paternità dell'opera e di condividerla allo stesso modo. La citazione di estratti è comunque sempre autorizzata, nei limiti previsti dalla legge. 


\title{
7/ Feminist sex wars
}

\author{
Arianna PASQUALINI
}

Con feminist sex wars si fa riferimento al dibattito interno al mondo femminista riguardo al modo in cui alcune pratiche sessuali possano o meno contribuire all'emancipazione femminile. Questioni come la prostituzione e la pornografia sono ancora centrali nel dibattito contemporaneo, che schiera da una parte le abolizioniste, le femministe anti-pornografia e dall'altra le femministe chiamate "pro-sex". Nel saggio che segue si analizzeranno le implicazioni e i dibattiti scaturiti intorno agli anni Ottanta, quando le prostitute cominciarono ad organizzarsi in movimenti per rivendicare la loro libertà di scelta e si coniò l'espressione sex work per riferirsi all'industria del sesso rigettando lo stigma sociale con la quale è stata spesso marchiata.

\section{Introduzione. Rivoluzione sessuale e fratture ideologiche}

Si sente spesso parlare di "morte del femminismo" da parte di chi critica quei cambiamenti che hanno snaturato la teoria femminista degli anni Sessanta e Settanta. In realtà si può forse parlare di un femminismo innovativo e post-moderno, identificabile come femminismo della terza ondata per distinguerlo dai precedenti ${ }^{1}$. Se infatti le sessantottine della seconda ondata si facevano portatrici del pensiero della differenza ${ }^{2}$ e si ponevano contro ogni forma di sfruttamento del corpo femminile, alle soglie del nuovo millennio ha preso piede una prospettiva più radicale che,

\footnotetext{
${ }^{1}$ Si può parlare di tre ondate del femminismo: la prima ondata risale a fine Ottocento-inizio Novecento e si propone principalmente il raggiungimento della parità giuridica e dei diritti fondamentali come il diritto al voto; la seconda ondata, nata tra gli anni Sessanta e Settanta, mira a riaffermare la specificità femminile e pone l'attenzione su temi nuovi quali la sessualità; la terza ondata, che può dirsi avviata a partire dagli anni Novanta, si pone come un femminismo radicale che tiene conto delle diversità, che decostruisce la nozione di genere e che affronta temi quali prostituzione e pornografia secondo una nuova prospettiva.

Cfr. SEVERGNINI, Chiara, «Dalle suffragette agli anni Novanta, breve storia del movimento femminista», in La Stampa Donne, 5 marzo 2016, URL:

<http://www.lastampa.it/2016/03/05/italia/cronache/speciali/festa-della-donna/dalle-suffragette dellottocento-agli-anni-breve-storia-del-movimento-femminista-J8I1IvCbVFEjK32xTSouzJ/pagina.html> [consultato il 24 agosto 2017].

${ }_{2}^{2}$ Per il pensiero della differenza si rimanda al secondo paragrafo.
} 
contro l'affermazione della specificità femminile, vuole invece tener conto delle soggettività individuali. Si tratta di un nuovo tipo di femminismo che lavora inoltre su identità sessuali dislocate, sul concetto di genere e di libera scelta, e si scaglia contro il determinismo biologico.

Se da una parte la suddivisione in ondate è da ponderare per la sua schematicità, può tuttavia risultare efficace per focalizzare il dinamismo del movimento ed affrontare la questione del dibattito interno al mondo femminista. A far luce su questo punto può essere interessante un saggio della giornalista americana Susan $\mathrm{Faludi}^{3}$, il quale mette in luce e denuncia quel conflitto generazionale tra donne che è in corso a partire dagli anni Novanta. Conflitto che, secondo Faludi, risulta essere il perfetto detonatore dell'autodistruzione del femminismo e che pone le giovani del nuovo millennio, accusate di essere donne "permale", in netto contrasto con il femminismo degli anni Settanta, additato invece come moralista. Le giovani di cui parla Susan Faludi, si fanno portatrici di un modello di femminilità nuovo, modulato nei termini della trasgressione, spesso percepita come priva di risvolti politici volti a stravolgere meccanismi di potere. Questo conflitto ingloba anche altre questioni, le quali hanno creato spaccature interne al mondo femminista, che, per semplificare, potrebbe essere distinto in due correnti: il femminismo detto "moralista" e il femminismo "sex-positive", chiamato provocatoriamente "femminismo puttana".

Il femminismo moralista va a criticare appunto i movimenti femministi delle ultime generazioni. Si tratta, secondo la definizione di Valeria Ottonelli, di:

\begin{abstract}
una posizione culturale e politica che, nel nome della libertà delle donne e della loro dignità, assume un atteggiamento sostanzialmente censorio, nei confronti degli uomini ma anche e soprattutto delle donne stesse. Secondo questo tipo di femminismo la liberazione delle donne deve avvenire attraverso una trasformazione intima di tutti i membri della società, che possa condurre ciascuno a capire quali sono i veri valori, il vero bene, il vero uso del proprio corpo, della propria sessualità e dei propri talenti. Nel fare questo si appella a un orizzonte simbolico e valoriale sostanzialmente conservatore e impone modelli di vita e di società che altro non sono se non rivisitazioni in chiave laica di vecchi miti familisti, religiosi e tradizionalisti ${ }^{4}$.
\end{abstract}

Valeria Ottonelli decostruisce i giudizi moraleggianti riguardanti il corpo delle donne e la sua mercificazione. La televisione italiana ad esempio, ritenuta portatrice di una visione gerarchica della società, mette in campo una rappresentazione mediatica di certi gruppi sociali in termini di subordinazione. Decretando la necessità di una maggior sensibilizzazione verso il tema e ponendo la questione mediatica in termini di mancata giustizia e irrispetto verso gruppi sociali come le

3 Cfr. FALUDI, Susan, «American Electra: feminism's ritual matricide», in Harper's magazine, 10/2010, pp. 2942. Il testo è stato tradotto in italiano con il titolo «La liberazione delle figlie», in Internazionale, 872, 12-18 novembre 2011, pp. 34-41. Cfr. anche CRISPINO, Anna Maria, «Prostitute, escort e donne permale», in Leggendaria, 85, 1/2011, pp. 6-7, in cui viene citato il saggio di Susan Faludi.

4 OTTONELLI, Valeria, La libertà delle donne. Critica al femminismo moralista, Genova, Il melangolo, 2011, p. 10. 
donne, Valeria Ottonelli ammonisce chi confonde la denuncia della subordinazione televisiva con quella di altri fenomeni culturali come ad esempio il giovanilismo e l'erotizzazione dell'iconografia femminile. Il rischio di un moralismo esteso a tutti i campi della cultura è notevole. Se da una parte, infatti, atteggiamenti moralisti sono mossi dalla volontà di reprimere comportamenti che potrebbero danneggiare terzi, dall'altra un giudizio morale privo di fondamenta critiche può esporre soggetti che già vivono in una condizione di marginalità alla disapprovazione e al disprezzo pubblico. Un'analisi, quella di Valeria Ottonelli, che offre un ottimo spunto per analizzare fenomeni sociali considerati generalmente in termini negativi come la prostituzione - un giudizio di valore che acuisce lo stigma di un gruppo sociale già debole.

In opposizione a questo tipo di orientamento femminista, ne emerge un altro che proclama la libertà di scelta e il diritto a una sessualità libera e svincolata dai giudizi morali altrui. Si tratta di un femminismo nato, intorno agli anni Ottanta, dall'aggregazione di gruppi di prostitute che decidono di prendere la parola per difendersi da chiunque intenda legiferare sulla loro vita imponendo doveri e non garantendo diritti. Come recita il manifesto dell'orgoglio delle sex workers francesi:

Femminismo puttana è opporsi alla sacralizzazione del sesso. Femminismo puttana è poter concepire e praticare la propria sessualità fuori dagli schemi della coppia, del matrimonio, dell'amore [...] Femminismo puttana è rendere la prostituzione un mezzo di crescita e di emancipazione delle donne [...] Lottare contro la puttanofobia significherà lottare contro il sessismo di fondo, lottare per poter essere, vestirsi, comportarsi da puttane...lottare, come tutte le donne, contro la paura di essere aggredite. Come dice il nostro slogan: dove, come, quando, quanto prendo, la scelta mi attiene, il corpo mi appartiene ${ }^{5}$.

Negli Stati Uniti le battaglie ideologiche che hanno creato tale frattura vennero chiamate ironicamente Sex Wars ${ }^{6}$. Ebbene, quando la sessualità divenne questione cruciale per il femminismo degli anni Sessanta e Settanta, si imbastì una riflessione teorica e si aprì il dibattito. Uno dei cambiamenti che scosse le coscienze femminili e che favorì la riflessione sulla questione $\mathrm{fu}$ forse la diffusione della pillola anticoncezionale, grazie alla quale il rapporto sessuale venne percepito non più solo funzionale alla procreazione, ma una ricerca continua del piacere fine a se stesso. La rivoluzione sessuale spinse molte donne alla scoperta della propria sessualità, non più

5 MAÎTRESSE NIKITA, SCHAFFAUSER, Thierry (a cura di), Fiere di essere puttane, Roma, DeriveApprodi, 2009, pp. 49-50.

${ }^{6}$ Cfr. ABRAMS, Kathryn, Sex Wars Redux: Agency and Coercion in Feminist Legal Theory, URL:

< http://scholarship.law.berkeley.edu/cgi/viewcontent.cgi?article=2734\&context=facpubs >, [consultato il 21 agosto 2017]; RUBIN, Gayle S., Thinking Sex: Notes for a Radical Theory of the Politics of Sexuality, in AGGLETON, Peter, PARKER, Richard (eds.), Culture, Society and Sexuality, London, Routledge, 2006, pp. 143178, URL: <http://citeseerx.ist.psu.edu/viewdoc/download?doi=10.1.1.462.7005\&rep=rep1\&type=pdf> [consultato il 21 agosto 2017]. 
repressa e sottomessa. La diffusione della pornografia, negli stessi anni, se da una parte favorì il superamento dei tabù, dall'altra, secondo alcune, avrebbe ridotto l'immagine della donna a oggetto sessuale.

La femminista Michi Staderini, analizzando le correnti estreme che hanno preso piede nel femminismo statunitense, menziona, da un lato Andrea Dworkin e Catherine Mc Kinnon, che definiscono la pornografia come la rappresentazione del potere maschile e misogino, dall'altro il collettivo Samois, il quale critica la visione romanticizzata del sesso portata avanti dal femminismo proibizionista antipornografico ${ }^{7}$. Staderini si pone al di sopra delle parti, precisando: «occorre criticare le forme di relazione tra i partners del rapporto sessuale, non i modi in cui si danno il piacere sessuale. Poiché è nel gestire la relazione che passa, a mio avviso, il potere nello specifico dei rapporti sessuali» ${ }^{8}$.

Vari fattori dunque contribuirono ad aprire il dibattito intorno al tema della sessualità. Tra questi appunto la diffusione della pornografia e la nascita di movimenti organizzati di prostitute e, con essa, la diffusione del termine sex work, che contribuì all'elaborazione di una diversa concezione di prostituzione, non più vista unicamente in termini di sfruttamento 9 .

\section{Lavoro sessuale in Italia: oppressione o espressione?}

Prendendo in considerazione ad esempio il contesto italiano, la spaccatura interna al movimento femminista emerge chiaramente. Qui, negli anni Settanta, sulla scia dell'interesse generale per gli scritti di Betty Friedan, nasce un nuovo tipo di femminismo. Sono anni di autocoscienza, di autoanalisi e di denuncia dell'oppressione maschile, ma anche di contestazione dell'educazione impartita dalle madri. Sono gli anni del separatismo, in cui le femministe vogliono portare avanti le loro battaglie per le donne tra donne. Mirano ad una rivoluzione del linguaggio che stravolga i canoni del patriarcato e che metta in campo una nuova teoria, quella della differenza, in cui la donna si riconosca e si definisca nel proprio "essere altro" dall'uomo.

Nasce il concetto di donnità, che consiste nella presa di coscienza dell'essenza femminile e nella riscoperta della propria autenticità, in netto rifiuto con ogni tentativo di descriversi in termini di complementarietà o di uguaglianza rispetto all'uomo. Si sente l'esigenza di riappropriarsi del

\footnotetext{
7 Cfr. STADERINI, Michi, Pornografie. Movimento femminista e immaginario sessuale, Roma, Manifestolibri, 1998.

8 Cit. in SARASINI, Bia, «Repressive o svergognate?», in Leggendaria, 122, 3/2017, pp. 10-11.

9 Per casi di sfruttamento e di tratta s'intende una realtà di lavoro forzato a cui non si acconsente ma che non si può rifiutare perché si è sotto minaccia. Spesso ad esempio i cosiddetti traffickers approfittano della situazione di persone che desiderano emigrare per sottoporle a violenze e a lavori forzati. Per questo argomento si veda GAROFALO GEYMONAT, Giulia, Vendere e comprare sesso, Bologna, Il Mulino, 2014, nel capitolo La tratta degli esseri umani.
} 
proprio corpo, di (ri)scoprire la propria femminilità. Si assiste insomma a una vera e propria rivoluzione culturale che mette in discussione i dogmi tradizionali sul corpo della donna ${ }^{10}$.

Le analisi femministe di questi anni muovono una critica all'oggettivazione della donna, madre e prostituta, figure entrambe condannate ad un'esistenza di schiavitù e subordinazione. In questi anni infatti, «il pensiero femminista tenta il superamento della dicotomia tra la funzione riproduttiva della donna-madre e la funzione genitale della donna-donna, che corrisponde sostanzialmente alla secolare contrapposizione madre/puttana» ${ }^{11}$. Le femministe operano dunque un duplice rifiuto: da una parte si rigetta la maternità come dovere istituzionalizzato, dall'altra si condanna la mercificazione del corpo femminile come necessaria per far fronte al diritto naturale dell'uomo di soddisfare le proprie pulsioni sessuali. L'obiettivo che si pongono ruota attorno alla questione dell'autodeterminazione, da raggiungere attraverso il rifiuto del ruolo di subalterne. «Nella figura della prostituta viene individuata l'espressione più evidente dell'oppressione patriarcale. La prostituta si presta in qualche modo a divenire oggetto sessuale, ad autoavvilirsi di fronte all'uomo»" ${ }^{12}$.

Le punte del femminismo più radicale propongono provocatoriamente l'introduzione del salario per il lavoro domestico, compreso il servizio sessuale nei confronti del marito, percepito anch'esso come una sessualità-dovere in cui rimane escluso il piacere sessuale della donna. Inoltre, lottano per un'abolizione totale della prostituzione, chiave di volta per un cambiamento della società e rovesciamento di quei ruoli socialmente imposti.

Negli stessi anni Ottanta nascono comitati di prostitute, i quali si pongono in netto contrasto con quei movimenti femministi che, tendendo alla ricerca della specificità femminile, si proiettano verso quel modello maschile che cercano loro stesse di distruggere. Viene dunque capovolta e smontata la critica femminista della donna-oggetto attraverso la proclamazione della libera scelta di utilizzare la propria sessualità in transizioni economiche e attraverso la rivendicazione di quel rapporto di scambio che è alla base del concetto di mercato: è proprio in forza dello scambio corpo-denaro che il rapporto prostituta-cliente non è un rapporto unilaterale di potere in cui la prostituta svolge un ruolo passivo.

All'interno di questo dibattito la prostituta si sente rifiutata dalle rappresentanti dello stesso sesso che mirano all'abolizione del suo lavoro. Vorrebbe ribaltare questa logica che la dipinge come vittima e proclamare la propria libertà di scelta. La condizione di sfruttate è un presupposto

\footnotetext{
${ }^{10}$ Per il femminismo italiano degli anni Settanta si veda: RIBERO, Aida, Una questione di libertà, il femminismo degli anni Settanta, Torino, Rosenberg \& Sellier, 1999; ID. (a cura di), Glossario. Lessico della differenza, Torino, Centro Studi e Documentazione Pensiero Femminista, 2007, URL:

<http://www.arcosricerca.it/Lavori/step/Glossario_Lessico\%20della\%20differenza_Regione\%20Piemonte.p df > [consultato il 24 agosto 2017]. Si veda in particolare la definizione di «Neofemminismo», pp. 172-179.

${ }^{11}$ SAPIO, Roberta, Prostituzione. Dal diritto ai diritti, Milano, leoncavallo libri, 1999, p. 88.

${ }^{12}$ Ibidem, p. 90.
} 
comune a tutte le donne: è questo il messaggio che vogliono far passare le prostitute e le femministe "sex-positive".

Il problema è non che il denaro crea la merce (ovvero l'oggetto sessuale); ma l'essere merce fa sì che si possa essere comprate. La prostituta che accede chiaramente al denaro tramite la vendita del proprio corpo è diversa dalla non prostituta solo perché fa un altro mestiere [...] Essere oggetto sessuale resta un dato comune a tutte le donne. È quindi questa condizione che va eliminata, non il mestiere della prostituta ${ }^{13}$.

Chiamare la prostituzione con il nome sex work è un atto significativo della presa di coscienza politica delle prostitute, che va maturandosi a partire dagli anni Ottanta. La nascita del Comitato dei Diritti Civili delle Prostitute (CDCP) nel 1982 a Pordenone, l'approvazione della Carta dei diritti di chi si prostituisce, le continue richieste di revisione della legge Merlin e l'elaborazione di progetti di prevenzione contro l'AIDS sono fattori sintomatici del clima di cambiamento di questi anni.

La proposta di legge «Abolizione della regolamentazione della prostituzione, lotta contro lo sfruttamento della prostituzione altrui e protezione della salute pubblica» fu avanzata da Lina Merlin nel 1948, approvata dieci anni dopo ed è in vigore tutt'oggi. Data l'impossibilità di eliminare la prostituzione, Lina Merlin si propose l'abolizione della regolamentazione e quindi il divieto di esercizio nelle case di tolleranza, come risultato di una lotta contro lo sfruttamento e contro il favoreggiamento della prostituzione. Il prodotto finale risulta essere un po' confuso in quanto configura la prostituzione come una realtà di fatto, ma non la proibisce né la consente. Con la legge Merlin si vieta il controllo diretto sulla prostituzione da parte dello Stato, ma anche da parte di soggetti privati e pubblici e quindi è resa perseguibile di sfruttamento e di favoreggiamento anche una qualsiasi persona che faciliti il lavoro di chi sceglie deliberatamente di prostituirsi ${ }^{14}$.

L'idea di base delle leggi abolizioniste è che la prostituzione sia «incompatibile con la dignità e il valore delle persone umane» (Preambolo alla Convenzione Onu del 1949). Ciò concretamente significa due cose: da una parte lo Stato sfavorisce la prostituzione e ne vieta l'organizzazione, la pubblicità, ecc., e dall'altra riconosce l'importanza di non criminalizzare e anzi proteggere le persone che si prostituiscono dagli abusi pubblici e privati, secondo l'idea che queste persone

\footnotetext{
13 STADERINI, Michi, «Denaro o no si resta oggetti», in Lucciola, 1, [1985?], pp. 4-5, cit. in SAPIO, Roberta, op. cit., pp. 96-97.

14 Per approfondimenti sulla Legge Merlin, cfr. SAPIO, Roberta, op. cit., pp. 81-82; GAROFALO GEYMONAT, Giulia, Vendere e comprare sesso, cit., capitolo Il mestiere più antico del mondo? - Legge Merlin.
} 
sono fondamentalmente vittime. Questo stato di vittime delle sex workers è centrale per capire la legge Merlin ${ }^{15}$.

Considerando dunque la prostituzione non come il frutto di una libera scelta ma unicamente come il prodotto di una realtà di sfruttamento - purtroppo presente anch'essa - la Legge Merlin pone divieti che, se non proibiscono l'atto della prostituzione, lo rendono praticamente impraticabile. Il CDCP invece inquadra la prostituzione non come un problema di ordine pubblico bensì come una questione sociale le cui radici sono da cercare nella costante domanda da parte dei clienti, «specchio di una società e della sua non-educazione sessuale, di una cultura maschilista che non pone su un piano paritario i rapporti uomo-donna» ${ }^{16}$.

La prostituzione è dunque, in questo caso, presentata come un fenomeno che attinge le sue ragioni d'essere nella società stessa, che al contempo la criminalizza e la stigmatizza come problema da risolvere e debellare. Se da una parte si propongono revisioni della legge Merlin, dall'altra si approva in tutto e per tutto l'abolizione delle case chiuse in quanto «luoghi tristi di schiavitù e sfruttamento voluti dallo Stato e sostenuti dalla morale della chiesa vaticana che ha sempre tollerato i bordelli purché non fossero vistosi» ${ }^{17}$. Quello che si propone è la rinuncia a una regolamentazione della prostituzione che costringerebbe le prostitute a rendersi riconoscibili, e l'avvio di pratiche che inducano un cambiamento culturale della società, germe appunto del bisogno della prostituzione. Solo attraverso la depenalizzazione della prostituzione si individuerebbero i casi di sfruttamento e di tratta e si concederebbe al contempo il rispetto dei diritti umani a chi si prostituisce per scelta.

\begin{abstract}
Noi prostitute siamo l'immagine della società che ci ha prodotto, ma che ci consente di esistere solo se funzionali ad essa. [...] La legge Merlin non ha risolto il problema, se di problema si tratta, della prostituzione e noi ne chiediamo la revisione; essa assieme al codice penale è complice di questa tragica situazione che fingendo di ignorare giuridicamente la prostituzione ne consente di fatto l'esistenza. Rivendichiamo inoltre il diritto ad usare e gestire il nostro corpo come più ci aggrada, in fabbrica come in strada, come donne, madri, sorelle, mogli, artiste, cittadine comunque della Repubblica Italiana ${ }^{18}$.
\end{abstract}

Il dibattito ruota dunque attorno a un principale interrogativo: può la prostituzione rappresentare un mezzo di emancipazione, o è piuttosto simbolo dell'oppressione patriarcale? Ad

\footnotetext{
${ }^{15}$ Ibidem.

${ }^{16}$ "Analisi sulla prostituzione e soluzioni possibili. Proposta politica. Pordenone 1994», in luccioleonline, URL: $<$ http://www.lucciole.org/content/view/11/3/>, [consultato il 24 giugno 2017].

${ }^{17}$ Ibidem.

18 «Le prostitute rivendicano il diritto all'esistenza», in luccioleonline, URL: <http://www.lucciole.org/content/view/5/3/>, [consultato il 24 giugno 2017].
} 
oggi si trovano associazioni e collettivi femministi che si proclamano ineluttabilmente contro il sistema prostituente in quanto portatore della cultura maschilista e violenta. Si pongono dunque anche contro ogni tentativo di utilizzare il termine "mercato" per definire tale sistema, in quanto banalizzerebbe una realtà di de-umanizzazione delle donne adottando un linguaggio che non è altro se non quello del capitalismo neoliberista. Non concepiscono la prostituzione come una libera scelta ma piuttosto come scelta in assenza di altre alternative e frutto di una cultura patriarcale e maschilista. Contro chi propone una legalizzazione della prostituzione, anche dunque contro Amnesty International, rispondono: «la precarietà, la globalizzazione, l'immigrazione non sono piaghe sociali che si risolvono vendendo donne sul mercato della schiavitù neo-liberista. [...] La prostituzione dei nostri corpi ci riguarda e mai permetteremo a voi di venderli per i vostri bisogni» ${ }^{19}$.

Un altro esempio di spaccatura interna al femminismo lo si può trovare nella storia del movimento Se non ora quando ${ }^{20}$, il quale, nato ai tempi del Berlusconismo, si opponeva alla cultura diffusa che legittimava comportamenti lesivi della dignità delle donne. Il movimento esortava tutte le donne a prendere coscienza di sé e a difendere il valore della propria dignità, a rifiutare quella rappresentazione delle donne come oggetto di scambio sessuale, proposta dai mass media. Come corollario dunque, se non ora quando, ha scarsa tolleranza nei confronti di quelle donne che rinunciano alla propria dignità per raggiungere mete importanti e/o per guadagno economico. Il movimento ha conosciuto una profonda divisione interna in merito a questioni di coscienza, come ad esempio la prostituzione, ma anche e soprattutto la maternità surrogata. È sempre stato un movimento composto da diverse anime, ricco di pluralità, tanto da arrivare alla frattura in Se non ora quando Factory e Se non ora quando Libere; quest'ultimo di recente ha chiesto la messa al bando della maternità surrogata in tutta Europa ${ }^{21}$.

In opposizione a questo femminismo abolizionista, è appena fiorito in Italia il movimento Non una di meno, incarnazione del femminismo di terza ondata e portavoce dello sciopero globale delle donne del Lotto Marzo 2017.

E il loro non è un femminismo "rassicurante" come poteva essere, per certi versi, quello di Se Non Ora Quando. Al contrario, è un femminismo radicale da un lato e molto più attento alle

\footnotetext{
${ }^{19}$ «Femministe precarie contro il sistema prostituente», in Resistenza Femminista, 28 maggio 2015, URL: <http://www.resistenzafemminista.it/femministe-precarie-vs-sistema-prostituente/>, [consultato il 24 giugno 2017].

${ }^{20}$ SAPPINO, Luca, "Cosa resta di Se non ora quando e perché le femministe si sono divise», in l'Espresso, 9 dicembre 2015, URL: < http://espresso.repubblica.it/attualita/2015/12/09/news/cosa-resta-di-se-non-oraquando-e-perche-le-femministe-si-sono-divise-1.242733?refresh_ce > [consultato il 24 giugno 2017].

${ }^{21}$ SAPPINO, Luca, SIRONI, Francesca, «Appello femminista contro l'utero in affitto. E c'è chi dice: “è una mossa sbagliata"», in l'Espresso, 04 dicembre 2015, URL: <http://espresso.repubblica.it/attualita/2015/12/04/news/Appello-femminista-contro-l-utero-in-affitto-ece-chi-dice-e-sbagliato-1.242261>, [consultato il 24 giugno 2017].
} 
diversità - etniche, di orientamento sessuale e di identità sessuale - dall'altra, che "osa" addirittura decostruire la nozione di genere, insomma è un femminismo intransigente e innovativo allo stesso tempo che difficilmente può scendere a compromessi ${ }^{22}$.

\section{La retorica abolizionista: il dibattito in Francia e il modello svedese}

La Francia ${ }^{23}$ abbandonò la via del regolamentarismo dopo un secolo e mezzo, nel 1946, con la legge Marthe Richard che decretò la chiusura delle case chiuse. Da allora si è avviato un processo di intimidazione delle prostitute che vede il suo apice con l'arrivo della destra al governo e la stipulazione della legge sulla sicurezza interna di Nicolas Sarkozy, nel $2003^{24}$. Questa legge nella teoria voleva combattere lo sfruttamento della prostituzione, nella pratica rispose alle esigenze della "gente per bene", eliminando il degrado dalle strade parigine e cacciando le immigrate clandestine. L'adescamento passivo venne reso perseguibile e passibile di un'ingente multa e due mesi di reclusione, fino a contemplare l'espulsione delle immigrate. Sarkozy accentuò la repressione poliziesca e lasciò la definizione di adescamento alla libera interpretazione della polizia. La logica conseguenza consistette, da un lato, in un aumento delle estorsioni, delle intimidazioni e delle violenze, anche sessuali, da parte della polizia e, dall'altro, in un aumento della clandestinità e del ruolo della malavita. Si moltiplicarono infatti i casi di sfruttamento e di tratta, poiché si preferiva lavorare sotto la protezione di un protettore piuttosto che in condizioni di violenza di Stato.

Sono nate dunque associazioni e movimenti per far fronte a questo tipo di soprusi, che in Francia si sono risolti in una vera e propria caccia alle prostitute. Già prima della legge Sarkozy, negli anni Settanta, la sessualità fu posta al centro della lotta delle donne e la prostituzione divenne tema su cui condurre una vera e propria riflessione teorica. Nel 1975 si assistette alla nascita del primo movimento francese di prostitute, il quale lanciò le prime proteste contro le politiche repressive del periodo e contro il progetto di legge che sarebbe stato approvato nello stesso anno. Questa legge prevedeva la condanna delle prostitute a multe molto alte e contemplava anche la possibilità di arresto. Non fece altro, insomma, se non istigare la polizia alla violenza e alimentare le casse dello Stato.

\footnotetext{
${ }^{22}$ GAIASCHI, Camilla, «Siamo alla terza ondata, il femminismo intransigente: contano i valori soggettivi non l'identità di gruppo", in Corriere della Sera, La 27esima ora, 4 marzo 2017, URL: <http://27esimaora.corriere.it/17_marzo_02/donne-sono-capaci-muoversi-unite-nome-liberta-dirittisiamo-terza-ondata-femminismo-intransigente-contano-valori-soggettivi-non-l-identita-gruppo-2bdd7e6cff65-11e6-9d9a-77b6de6db49f.shtml> [consultato il 25 agosto 2017].

${ }^{23}$ Il caso francese sarà analizzato facendo riferimento principalmente a MAÎTRESSE NIKITA, SCHAUFFAUSER, Thierry (a cura di), op. cit.

${ }^{24}$ Cfr. ibidem, pp. 23-26.
} 
Comunque, grazie al consenso che guadagnò il movimento negli anni Settanta e Ottanta, un gran numero di prostitute rinunciò al protettore e iniziò a lavorare in proprio. Anche in Francia, dunque, la prostituta prese coscienza di sé e divenne soggetto politico attivo e combattivo, pronto a denunciare qualsiasi tipo di violenza. Il movimento delle prostitute iniziò dunque ad organizzarsi a livello mondiale e vide lo svolgersi del primo congresso internazionale delle prostitute, ad Amsterdam nel 1985, durante il quale venne redatta la Carta internazionale delle prostitute. Come già accennato, gli anni Ottanta furono gli anni delle Sex Wars, che portarono ad una frattura del movimento femminista. Per quanto riguarda il caso francese i movimenti femministi non furono alleati del movimento delle prostitute, ma sempre divisi tra loro sulla questione, concordi solo sulla chiusura delle case chiuse e sull'opposizione alle politiche repressive.

A partire dagli anni Novanta diventò maggioritaria quella linea del femminismo orientata a posizioni abolizioniste molte rigide, soprattutto con la creazione del Collectif National pour le droits des femmes (CNDF), fondato nel 1996. Questo collettivo raggruppa associazioni femministe, sindacati e partiti politici di sinistra e rappresenta la linea di tendenza del movimento femminista ufficiale, che se formalmente si propone come scopo l'uguaglianza tra i sessi, di fatto non ha fatto che favorire la promulgazione di leggi discriminatorie, come la Lsi di Sarkozy e la legge che proibisce simboli religiosi a scuola. Il collettivo trova la sua voce nell'associazione Ni Putes ni soumises, la cui logica opera "contro le donne che non corrispondono al modello occidentale e giudeo cristiano di donna emancipata» ${ }^{25}$. Quindi contro le donne musulmane che indossano il velo, considerato dal movimento come simbolo di sottomissione anziché simbolo di resistenza e di affermazione in una società francese razzista, e contro le prostitute, non riuscendo a concepire la prostituzione come un mezzo di emancipazione utile per liberarsi dall'asservimento a un potere.

Per contro, nel 2006, sulla scia dell'entusiasmo suscitato dalla "Conferenza europea delle lavoratrici del sesso" tenutasi a Bruxelles per la ratifica della Dichiarazione dei diritti delle lavoratrici del sesso, nasce l'associazione Les Putes, fondata da Thierry Schauffauser e Maîtresse Nikita, autori del libro Fiere di essere puttane. Les Putes fa tesoro della militanza di Act up-Paris ${ }^{26}$, aggiungendovi un approccio innovativo che si propone di combattere il marchio d'infamia legato alla prostituzione. Les Putes rappresenta una novità rispetto ai movimenti nati nel 1975, raccogliendo le eredità e le speranze comuni a tutte le associazioni e sentendo forte la necessità di «trasporre il modello di attivismo della lotta dei froci-sieropositivi alle lavoratrici del sesso» ${ }^{27}$. Non avendo

\footnotetext{
${ }^{25}$ Ibidem, p. 59.

${ }^{26}$ Aids coalition to unleash power, associazione nata nel 1989, impegnata nelle problematiche relative all'omofobia, alla prevenzione e alla lotta contro l'Aids.

${ }^{27}$ MAÎTRESSE NIKITA, SCHAUFFAUSER, Thierry (a cura di), op. cit., p. 14.
} 
ricevuto alcun appoggio da parte di associazioni femministe, né da parte della sinistra francese, l'associazione punta all'autorganizzazione lanciando Pute pride e azioni di militanza attiva.

Le militanti di Les Putes non si definiscono nemmeno regolamentariste poiché contro alle proposte di riapertura delle case chiuse. Si ripropongono di combattere la legge di sicurezza, lo stigma, e quella che chiamano puttanofobia, una discriminazione ben radicata nella nostra cultura. Contro la visione bigotta della sessualità che viene impartita dai piani alti, rivendicano la propria intelligenza e il diritto di autodeterminazione, reclamano il proprio ruolo di attrici attive, dichiarando di non vendere stupidamente il proprio corpo, ma di proporre servizi sessuali. Sono contrarie a ridurre il corpo delle donne alla funzione sessuale: la prostituta che mette a disposizione il proprio sesso è paragonabile al lavoratore che mette a disposizione la propria forza lavoro. Ritengono che ogni lavoro sia al contempo una forma di violenza e un mezzo di emancipazione: «ciò che bisogna capire è che la violenza esiste più facilmente nei mestieri più stigmatizzati, proprio perché non riconosciuti. La schiavitù non si combatte quindi negando la realtà di un lavoro, ma concedendo diritti ai lavoratori che lo esercitano» ${ }^{28}$. Le attiviste di Les Putes condannano ogni tipo di negazione della loro libertà di scelta, sia che provenga da protettori che da abolizionisti. Vedono l'abolizionismo come una forma di maltrattamento teorico, che minimizza e considera le prostitute vittime incapaci di scelte consapevoli. Rigettano il termine "vittima" e si riappropriano impavide dell'insulto "puttana", operando anche in questo caso una rivoluzione del linguaggio.

\footnotetext{
Rovesciando l'insulto, usiamo il termine "puttana" per esprimere orgoglio per ciò che siamo oppure utilizziamo "lavoratrici del sesso", poiché esprime chiaramente l'idea che la prostituzione sia un lavoro e che debba quindi essere riconosciuto come tale, con ciò che comporta in termini di diritti. [...].

Dobbiamo essere capaci di autodefinirci attraverso l'insulto di puttane. Il fatto di dichiararsi tali annulla il risvolto stigmatizzante del termine, che così non è più un insulto, ma diventa l'indicatore di un'identità di cui essere orgogliose $\mathrm{e}^{29}$.
}

Combattono quindi la condizione di isolamento sociale a cui sono sottoposte, attraverso la costruzione di una forte identità politica di resistenza contro le norme sessiste. Vogliono combattere il modello di femminilità portato avanti dagli stessi femminismi del passato, che avevano escluso la componente lesbica dal movimento. Cercano continuamente il loro sostegno in quanto ritengono che la lotta delle prostitute debba essere lotta di tutte le donne, contro qualsiasi

\footnotetext{
${ }^{28}$ Ibidem, p. 70.

${ }^{29}$ Ibidem, pp. 65, 73.
} 
limitazione della libertà sessuale. Accusano le femministe abolizioniste di incoraggiare lo stigma e il sessismo che dicono di voler combattere.

Pensiamo che la lotta delle prostitute sia una lotta femminista. Sfortunatamente, fino ad oggi, siamo state escluse dalla maggior parte di questi movimenti. La nostra esclusione è frutto di un malinteso: la maggior parte delle femministe ci considera vittime della prostituzione, mentre noi ci consideriamo vittime delle cattive condizioni in cui lavoriamo. [...].

Smettetela di pensare che non possiamo essere femministe come voi. Contribuite a farci passare per dei corpi che si vendono e così incoraggiate le violenze sessiste contro di noi ${ }^{30}$.

Vogliono sfatare i luoghi comuni che attribuiscono alle prostitute un profilo psicologico, un'infanzia problematica, traumi dell'inconscio che le avrebbe spinte ad intraprendere questa scelta. Contro chi ritiene che tale scelta conservi un ordine sessuale patriarcale rispondono: «La sessualità traduce rapporti di potere. L'intervento del denaro può essere considerato come fattore di consolidamento o di rovesciamento di tale potere» ${ }^{31}$.

Un'altra voce fuori dal coro è quella di Morgane Merteuil, giovane donna, attivista, segretaria generale dello Strass $^{32}$, il sindacato francese del lavoro del sesso nato nel marzo del 2009. Nel suo pamphlet Libérez le féminisme $!^{33}$ si scaglia contro le femministe abolizioniste portando avanti la sua idea di un femminismo prosex. Venticinquenne femminista, la Merteuil abbandonò il lavoro di cameriera, mal retribuito, per prendere volontariamente la strada della prostituzione. Critica quel femminismo che dovrebbe essere sinonimo di libertà ma che non fa altro che imporre «un'unica $\mathrm{e}$ sola immagine della donna, mainstream e borghese $»^{34}$. La propaganda delle associazioni criticate dalla Merteuil come l'associazione Ni putes ni soumises ruota attorno al principio incontrovertibile della dignità della donna e ne erige un solo modello di cui tutte dovrebbero far parte. La Merteuil ribatte proponendo un «femminismo plurale che ammetta, riconosca e difenda anche altri modi di emanciparsi. Non tutte le donne aspirano necessariamente alle stesse cose, alcune, ad esempio, amano il sesso. E sì, prostituirsi può essere un modo di riappropriarsi della propria sessualità e del proprio corpo» ${ }^{35}$.

\footnotetext{
${ }^{30}$ Ibidem, pp. 60, 74 (lettera aperta alle nostre sorelle femministe, 8 marzo 2006).

${ }^{31}$ Ibidem, p. 82.

${ }^{32}$ Syndacat du Travail Sexuel.

${ }^{33}$ MERTEUIL, Morgane, Libérez le féminisme!, URL:

<http://www.l-editeur.fr/sites/default/files/EXTRAIT-LLF.pdf > [consultato il 21 agosto 2017].

${ }^{34}$ CARDINALE, Alessandra, «Francia, il femminismo pro-sesso, pro-porno, pro-puttane di Morgane Merteuil», in Il Fatto Quotidiano, 28 settembre 2012,

URL: <http://www.ilfattoquotidiano.it/2012/09/28/francia-femminismo-pro-sesso-pro-porno-pro-puttanemorgane-merteuil/357507> [consultato il 26 giugno 2017].

${ }^{35}$ Ibidem.
} 
Definisce la prostituzione come una forma di emancipazione, ovviamente relativa alle condizioni in cui si esercita e in cui si è scelto di farlo. Lotta contro l'abolizionismo in quanto posizione paternalista che rende il lavoro delle prostitute ancora più pericoloso. Si batte per l'abrogazione del reato di adescamento passivo e per il diritto di esistere in quanto individuo che può disporre liberamente del proprio corpo, cosicché ogni donna possa essere rispettata indipendentemente dalla scelta che decide di fare. Oggi si dedica esclusivamente alla militanza e al sindacato, luogo di elaborazione di un modello sessuale autogestito e libero, nel quale si combatte da anni contro lo sfruttamento della prostituzione e contro la tratta di esseri umani.

Le battaglie attuali promosse dallo Strass ${ }^{36}$ mirano, tra le altre cose, a protestare contro la legge che è entrata in vigore in Francia nel 2016 e che segue il modello svedese, basato sulla criminalizzazione dei clienti. La Svezia, infatti, partendo dal presupposto che la prostituzione femminile sia un caso eclatante di violenza di genere, ha elaborato la propria legislazione al riguardo sulla base di questo precetto. Lo ha fatto attraverso la perseguibilità dei clienti: per ridurre il mercato del sesso nelle strade senza colpire direttamente le vittime, si mira a colpire la domanda e dunque punire i clienti ${ }^{37}$. Tale legge, chiamata Kvinnofrid, "della pace delle donne", è il risultato di studi che hanno preso piede verso la fine degli anni Settanta e che hanno portato ad un mutamento di prospettiva nei confronti del fenomeno della prostituzione, visto ora come una questione di politiche di genere. Si tratta di una legislazione, dunque, che si erge a paladina della giustizia ponendosi alti obiettivi. Ma, «anche se la legge teoricamente punta a proteggere le sex workers, è evidente che lo scopo principale è quello di proteggere la società da quella che è percepita come una malattia sociale $»^{38}$. La legislazione svedese insomma ha suscitato aspri dibattiti e ha sollevato un po' ovunque un certo interesse, in quanto considerata spesso la soluzione al "problema" della prostituzione: colpire il cliente, ridurre la domanda, eliminare la prostituzione e dunque "salvare" le vittime di sfruttamento.

Dagli scritti di Petra Östergren ${ }^{39}$, antropologa sociale di Stoccolma che si occupa di analizzare il pensiero dei movimenti anti-porno e anti-prostituzione, emerge come la prostituzione in Svezia

\footnotetext{
${ }^{36}$ Per ulteriori informazioni sulle battaglie condotte dallo Strass si veda direttamente il sito del sindacato, URL: < http://strass-syndicat.org/en/ > [consultato il 24 agosto 2017].

${ }^{37}$ Dunque, in Svezia è legale vendere servizi sessuali ma è illegale comprarli: l'acquisto di servizi sessuali da parte di un uomo nei confronti di una donna è punito con la detenzione in carcere del cliente (oppure, col pagamento di un'ingente multa, se il cliente ammette la colpa). Cfr. GAROFALO GEYMONAT, Giulia, «La prostituzione tra abolizionismo, proibizionismo e legalizzazione -Punire i clienti: una nuova soluzione?», in Micromega,5/2014, pp. 66-78; ID., «Il lavoro sessuale in Europa», in Il Mulino, 2/2011, pp. 291-299, p. 295.

${ }^{38}$ «Le bugie sul sex work in Svezia», in luccioleonline, URL: <http://www.lucciole.org/content/view/160/3/> [consultato il 27 giugno 2017].

${ }^{39}$ ÖSTERGREN, Petra, Poor, horor och Feminister, Stockholm, Natur och Kultur, 2006 (in cui documenta le esperienze e le opinioni delle sex workers); DODILLET, Susanne, ÖSTERGREN, Petra, «The Swedish Sex Purchase Act: Claimed Success and Documented Effects», Conference paper presented at the International Workshop: Decriminalizing Prostitution and Beyond: Practical Experiences and Challenges. The Hague, 3-4 marzo 2011, URL: <http://www.plri.org/sites/plri.org/files/Impact\%20of\%20Swedish\%20law0.pdf> [consultato il
} 
venga percepita come malattia sociale e forma di violenza maschile, come fenomeno che va abolito per la creazione di una società più equa. Molte lavoratrici del sesso ritengono che la legge contro l'acquisto dei servizi sessuali sia contraddittoria e discriminatoria. Gli effetti della legge, inoltre, risultano essere negativi in quanto è cresciuta la possibilità di avere a che fare con clienti pericolosi. Inoltre, la riduzione del numero dei clienti e il conseguente aumento della competizione tra prostitute hanno provocato l'abbassamento dei prezzi e l'esposizione delle prostitute alla strada. Un altro effetto negativo denunciato dalle sex workers consiste nel trattamento sociale discriminatorio e nella stigmatizzazione alla quale sono continuamente sottoposte. Si sentono private di potere decisionale, si sentono vittime non del sistema prostituente ma di un sistema legislativo per niente democratico, si sentono abbandonate dalle stesse autorità che dicono di volerle proteggere. Non sentono il bisogno di nessun tipo di rieducazione in quanto non vedono nel loro lavoro nulla di intrinsecamente sbagliato o deviante. Dichiarano inoltre di sentirsi strumentalizzate dai media, dai politici, e dalle stesse femministe. Tra le femministe che aderiscono alla corrente abolizionista spicca Kaisa Ekis Ekman, giornalista svedese autrice di Varat och varan: Prostitution, surrogatmödraskap och den delade människan ${ }^{40}$. In Varat och Varan, pubblicato nel 2010, l'autrice si propone di formulare una teoria contro la prostituzione e contro altre tematiche come la maternità surrogata. Contro chi giustifica tali pratiche, contro le rappresentazioni mediatiche della prostituzione che la dipingono come carriera glamour e intelligente, contro chi si batte per l'abolizione della legge svedese, Kajsa Ekis Ekman risponde in virtù della sua impostazione femminista e marxista, proclamando l'incompatibilità tra prostituzione e uguaglianza tra i sessi. La prostituzione sarebbe «nemica della liberazione sessuale, del desiderio reciproco, del piacere condiviso ${ }^{41}$. Critica quel femminismo che si è lasciato convincere dalla teoria secondo la quale la prostituzione coinciderebbe con il raggiungimento di una maggiore libertà del proprio corpo. Infatti:

Il discorso in favore del lavoro sessuale cerca di appropriarsi dei temi centrali di tutte le ideologie per infiltrarsi nelle sfere della società. Ha la proprietà incredibile di combinare l'idea di rivolta (gli oppressi e le oppresse contro il potere) con il capitalismo (la libertà di vendere). La prostituzione è circondata da miti che ci impediscono di vedere la tragedia che suppone che un essere umano ne compri un altro, un essere umano che riduce l'altro in uno stato di oggetto, di merce $^{42}$.

\footnotetext{
25 agosto 2017].

${ }^{40}$ EKMAN, Kajsa Ekis, Varat och varan: Prostitution, surrogatmödraskap och den delade människan, Stockholm, Leopard, 2010.

${ }^{41}$ CASAS VILA, Glòria, «La prostituzione è nemica della liberazione sessuale», in Il Corsaro, 7 gennaio 2015, URL: <http://www.ilcorsaro.info/altrove-2/la-prostituzione-e-nemica-della-liberazione-sessuale-intervistaa-kajsa-ekis-ekman.html> [consultato il 30 giugno 2017].

${ }^{42}$ Ibidem.
} 
In Varat och Varan, Kajsa Ekis Ekman parla di dissociazione nella prostituzione come strategia di autodifesa per cui la prostituta, durante il lavoro sessuale, è costretta ad estraniarsi da se stessa per riuscire a gestire la sua prostituzione. Si tratterebbe di una sorta di strategia di sopravvivenza in cui avviene dunque la scissione tra corpo e mente. Questo renderebbe il lavoro sessuale fonte di dissociazione e di reificazione, privandolo di ogni valore e contenuto. Lo stesso Marx ha utilizzato il termine "reificazione" per indicare il processo in cui, nel sistema capitalistico, il lavoro umano diventa semplice attributo della cosa che si mira a produrre. Kajsa Ekis Ekman critica i tentativi di ridefinizione del termine "prostituta" che mirano ad abolire il termine "vittima" per sostituirlo con sex worker in modo da conferire al soggetto capacità di agire e di autodeterminarsi. Secondo la femminista, invece, chi mira all'abolizione del termine "vittima" avrebbe una visione cinica della società, in cui ogni individuo è responsabile della propria condizione, qualsiasi essa sia. Negare il termine "vittima" significherebbe negare un sistema di ingiustizie e annullare le differenze di potere: «facendo della vittima un tabù, si possono legittimare le disuguaglianze di classe e la discriminazione di genere, perché senza vittima, non c'è colpevole»» ${ }^{43}$.

È sbagliato secondo lei considerare fenomeni quali la prostituzione come scelte che hanno un potenziale sovversivo. L'errore stesso della sinistra secondo Ekis Ekman è stato quello di rinunciare a cambiare radicalmente la società per giungere ad un mutamento della prospettiva e ad una ridefinizione dello status quo. La femminista svedese guarda con ammirazione la legge svedese, sostenendo che essa non voglia affatto depenalizzare la prostituzione, ma responsabilizzare il cliente, eliminando il vero problema dell'atto della prostituzione. Convinta che non si possa avere un'industria del sesso senza una tratta di persone, crede che l'eliminazione della prostituzione sia l'unica soluzione e auspica che la legge svedese venga estesa a tutta Europa.

\section{Conclusioni. Sessualità e potere}

Le sex wars hanno dunque creato una strappo ideologico destinato inevitabilmente a rimanere scucito, la cui radice si potrebbe forse cercare all'interno di meccanismi di potere, i quali «intervengono modellando un sapere, una conoscenza dei fatti e stabilendo una verità ${ }^{44}$. Siano questi meccanismi di tipo istituzionale, religioso, o sociale e politico, da sempre si è tentato di

\footnotetext{
${ }^{43}$ ROSSI Maria, «Prostituzione, l'abolizione della vittima e la difesa postmoderna dello status quo», in info sulla prostituzione, 07 giugno 2014, URL:

<http://infosullaprostituzione.blogspot.de/2014/06/prostituzione-labolizione-della-vittima.html> [consultato il 30 giugno 2017].

${ }^{44}$ SUGAMELE, Laura, «La sessualità tra meccanismi di potere e controllo nel pensiero di Michel Foucault», in Rivista di scienze Sociali: Erottica, sguardi obliqui di corpi dilatati, 15, 2016, URL: $<$ http://www.rivistadiscienzesociali.it/la-sessualita-tra-meccanismi-di-potere-e-controllo-nel-pensiero-dimichel-foucault/> [consultato l'8 agosto 2017].
} 
definire il labile confine tra razionale e irrazionale, tra lecito e illecito, tra concesso e proibito, in ogni ambito, dunque anche quello sessuale. Questo è avvenuto ad esempio attraverso i precetti della morale cristiana, oppure attraverso le teorie freudiane, insomma attraverso un qualsiasi tipo di filtro che ha cercato di interpretare la sessualità normalizzandola in un unico modello socialmente accettabile. Seguendo la riflessione di Foucault presente nella sua Storia della sessualitá ${ }^{45}$, emerge come da sempre il potere normativo abbia cercato di disciplinare ogni tipo di comportamento allineandolo al modello prestabilito. Il potere repressivo punisce e sanziona i comportamenti considerati devianti e, nella sua forma disciplinare, riesce a modellare e delineare comportamenti e identità. Di conseguenza, ogni comportamento sessuale non normato viene considerato patologico e pericoloso per l'equilibrio sociale.

Per Foucault il corpo non è "sessuato» in alcun senso significativo prima di essere determinato entro un discorso che lo investe dell'«idea» di sesso naturale o essenziale. Il corpo acquisisce significato all'interno del discorso solo nel contesto delle relazioni di potere. La sessualità è un'organizzazione storicamente specifica del potere, del discorso, dei corpi e dell'affettività. In questo senso la sessualità è intesa da Foucault come ciò che produce il «sesso» in quanto concetto artificiale che effettivamente estende e maschera le relazioni di potere che sono responsabili della sua genesi ${ }^{46}$.

È in quest'ottica che si inserisce il dibattito attorno alla definizione di concetti quali identità di genere e lavoro sessuale, concetti continuamente sottoposti a meccanismi di potere e di conseguenza a convinzioni socialmente radicate. Come conclude Gayle Rubin nel saggio «Thinking sex»:

Like gender, sexuality is political. It is organized into systems of power, which reward and encourage some individuals and activities while punishing and suppressing others. Like the capital organization of labour and its distribution of rewards and powers, the modern sexual system has been the object of political struggle since it emerged and as it has evolved ${ }^{47}$.

\footnotetext{
${ }^{45}$ FOUCAULT, Michel, Storia della sessualità, 3 voll., Milano, ES, 2014-2015; Cfr. BUTLER, Judith, Questioni di genere, Il femminismo e la sovversione dell'identità, Roma-Bari, Laterza, 2013, edizione digitale, passim (in particolare da p. 104).

${ }^{46}$ BUTLER, Judith, op. cit., p. 105.

${ }^{47}$ RUBIN, Gayle, op. cit., p. 171.
} 


\section{L'AUTORE}

Arianna PASQUALINI ha studiato Lettere Moderne presso l'Università di Bologna, dove ha conseguito la laurea triennale nel 2016 con una tesi dal titolo Alterità africane nellimmmaginario occidentale del secondo Novecento: voci di una letteratura italiana tra coloniale e neocoloniale. Iscritta a Studi orientali nello stesso ateneo, attualmente partecipa al Master in African Studies presso la Dalarna University a Falun, in Svezia.

URL: < http://www.studistorici.com/progett/autori/\#Pasqualini > 